\title{
On the Use of Soy Protein in Diets for Juvenile Birds
}

\author{
Rebecca S. Duerr, DVM, MVPM, and Kirk C. Klasing, PhD \\ University of CALIFORNIA, DAVIS \\ DAVIS, CALIFORNIA
}

\section{INTRODUCTION}

Soy protein is commonly used by commercial animal food manufacturers as an inexpensive source of high quality protein, and when appropriately supplemented with methionine, has an amino acid profile that approaches whole egg. However, the use of soy products in baby bird diets has long been controversial in the rehabilitation community. There are several legitimate nutritional concerns about the use of soy that will be addressed in this article after a brief review of protein structure and function.

\section{ABOUT PROTEINS}

Proteins comprise the majority of an animal's body, and are especially enriched in muscles, tendons, and ligaments. They form important blood proteins like hemoglobin and albumin, plus they form a vast diversity of enzymes that facilitate the complicated chemical reactions that occur in the body. They are used to make hormones such as insulin, thyroid hormone, and adrenaline. Proteins are composed of hundreds to thousands of amino acids linked together. The identity and function of proteins are determined by the sequence and three-dimensional characteristics of the amino acid chain(s). Most animal protein is composed of 20 different amino acids. Species vary in whether they are able to make individual amino acids from

\footnotetext{
Rebecca Duerr is an avian veterinarian currently pursuing a $\mathrm{PhD}$ on the critical care nutrition of faunivorous seabirds with Dr. Klasing at University of California, Davis. She co-edited and authored four chapters in Hand-Rearing Birds (WileyBlackwell, 2007).
}

Kirk Klasing is a professor in the Animal Science Department at University of California, Davis. He primarily studies the interactions of nutrition and immunity in diverse avian species. He also serves as a nutritional consultant to numerous zoos, bird food manufacturers, and avian conservation projects worldwide. He is the author of Comparative Avian Nutrition (CAB Publishing, 1998). constituent parts carbon, nitrogen, oxygen, hydrogen, and sulfur. If an animal cannot make a particular amino acid, it is required in the animal's diet and is referred to as an essential amino acid. In avian species that have been studied, adult birds have 9 essential amino acids but growing chicks have 12. Physiologic states such as illness can raise the demand for certain amino acids in the diet because an animal might be unable to keep up with needs, even of some nonessential amino acids.

Animal tissues being used as food for other animals have full complements of amino acids and are considered to be complete proteins. Plant source proteins typically have lower levels of one or more essential amino acids, commonly methionine, lysine, or cysteine, and are considered incomplete proteins. Body tissues only can be constructed when all required amino acids are present. If one or more are missing, that tissue cannot be built until more of the limited amino acid becomes available. Individual amino acids may be added to deficient diets in order to alter the relative amount of amino acids to each other. In this manner, a plant source protein may be used to create a food that has a meat protein amino acid profile, such as one closely resembling whole egg.

\section{HYDROLYZED PROTEINS}

Hydrolysis is the process in digestion or manufacturing where water is used to break chemical bonds and break up a molecule such as a protein into smaller pieces. When a protein is thus broken down into smaller segments called polypeptides, it is essentially pre-digested and can be an excellent and highly bioavailable protein source for ill or very young animals. Hydrolyzed protein has been fragmented to the point where its initial source material is no longer relevant. When hydrolyzed, an amino acid is an amino acid regardless of parentage. Hydrolyzed soy protein is one such product. 


\section{PHYTOESTROGENS}

Phytoestrogens are molecules produced by plants that may exert estrogen-like reproductive effects on animals consuming them. Soybeans contain many bioactive compounds including several isoflavones, such as genistein and daidzein, which are phytoestrogens. This is considered a good thing in human nutrition. There are concerns that feeding high levels of isoflavones to growing chicks could alter levels of male or female hormones and thus alter sexual development, particularly for male chicks. As a result of this, it would not be prudent to feed soy products containing significant amounts of isoflavones to wild species where the effects on reproductive development have not been studied. The method by which soybeans are processed markedly affects their isoflavone level. In the process of isolating the protein from the soybean seed, virtually all of the isoflavone can be removed if desired. Manufacturers offer products that are high in isoflavones for human nutrition, but they also offer products that are very low. Hydrolyzed soy protein used in bird foods has levels of isoflavones that are extremely low and not of concern.

\section{ELECTROLYTES}

Depending on manufacturing methods, soy protein products can have excessive or miniscule amounts of sodium, potassium, or chloride in them. These minerals are important electrolytes in the body and help maintain appropriate water and $\mathrm{pH}$ balance. These electrolytes must be provided in the diet at sufficient levels and also in the correct proportions (balance). The nutritionist designing the diet needs to select soy protein sources that have suitable levels of these electrolytes and optimize their balance by supplementing additional amounts as needed to meet the bird's nutrient needs. When this is done correctly, soy protein can contribute an excellent electrolyte profile to the diet.

\section{CALCULATION OF CALCIUM PHOSPHORUS RATIOS (CA:P)}

When soy or other plant protein sources are used in diets, it complicates calculation of Ca:P, which is of particular importance to normal growth of the baby bird skeleton. This is because plant protein sources often have a significant portion of the total phosphorus bound to a chemical called phytate, which renders the phosphorus much less bioavailable. A portion, usually around one-third, of the phosphorus is not bound to phytate and is referred to as non-phytate phosphorus. This non-phytate phosphorus is digested and used at a rate similar to phosphorus in meat so non-phytate phosphorus is used for calculating the 'available' phosphorus in a diet. The best ratio of calcium to available phosphorus for a growing bird is about two to one. Consequently, if a diet containing both meat and plant protein has a nutritional analysis that only shows total phosphorus but not non-phytate phosphorus, it is impossible to calculate $\mathrm{Ca}: \mathrm{P}$ ratio from the given information since the list of ingredients on a label does not specify how much of the phosphorus comes from plant sources. This is not a concern with meat-only protein sources where the total calcium and total phosphorus amounts are straightforward. If a diet contains only plant sources of phosphorus, the Ca:P ratio should be much lower than two to one because the nutritionist that formulated the diet will have considered only the non-phytate portion of the total phosphorus.

\section{CONCLUSION}

Some soy products may contain potentially detrimental levels of phytoestrogens that may affect sexual development in growing chicks. However, hydrolyzed soy protein contains very little of these compounds. Avian nutritionists often need to adjust the amount of electrolytes in diets containing soy, and adjust the amount of calcium to balance the amount of bioavailable phosphorus. Overall, soy protein, particularly hydrolyzed soy protein, is an acceptable protein source for growing birds if it is properly supplemented with methionine. (

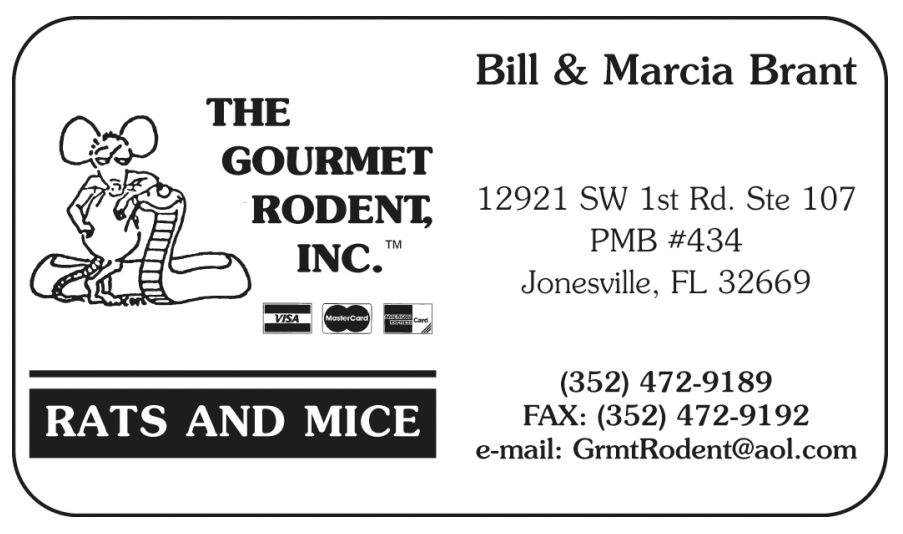

